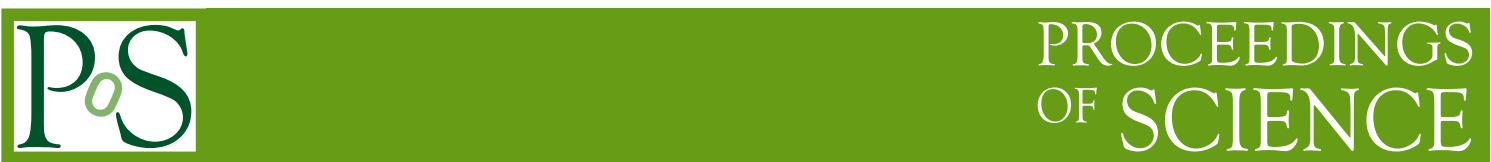

\title{
Constraints from electromagnetic cascades in the
} EBL

\section{Kachelrieß}

Institutt for fysikk, NTNU, Trondheim, Norway

High energy particles interacting with the extragalactic photon background initiate electromagnetic pair cascades. I discuss here the resulting constraints on UHECR models, the flux of cosmogenic neutrinos and the extragalactic magnetic field (EGMF). In the former case, the diffuse isotropic gamma radiation measured by Fermi-LAT is used to show that cosmogenic neutrino fluxes are only marginally detectable by existing and currently planned neutrino experiments. In the latter case, the non-observation of 1ES $0229+200$ by Fermi-LAT requires that the EGMF is stronger in at least $60 \%$ of space than $\sim 5 \times 10^{-15} \mathrm{G}$ (stationary source) or $\sim 10^{-17} \mathrm{G}$ (flaring source). Thus the (non-) observation of $\mathrm{GeV}$ extensions around $\mathrm{TeV}$ blazars probes the EGMF in voids and puts strong constraints on the origin of EGMFs, favoring a primordial origin.

25th Texas Symposium on Relativistic Astrophysics - TEXAS 2010

December 06-10, 2010

Heidelberg, Germany 


\section{Introduction}

The Universe is opaque to the propagation of $\gamma$-rays with energies in the $\mathrm{TeV}$ region and above. Such photons are absorbed by pair production on the infrared/optical extragalactic background light (EBL), initiating electromagnetic cascades in the intergalactic space, via the two processes $\gamma+\gamma_{b} \rightarrow e^{+}+e^{-}$and $e^{ \pm}+\gamma_{b} \rightarrow e^{ \pm}+\gamma[1]$. The cascade develops very fast until it reaches the pair creation threshold at $s_{\min }=4 E_{\gamma} \varepsilon_{\gamma}=4 m_{e}^{2}$, where $\varepsilon_{\gamma}$ is the typical energy of the background photons $\gamma_{b}$. Electrons continue to scatter on cosmic microwave photons in the Thomson regime with an interaction length around $5 \mathrm{kpc}$, producing photons with average energy

$$
E_{\gamma}=\frac{4}{3} \frac{\varepsilon_{\gamma} E_{e}^{2}}{m_{e}^{2}} \approx 100 \mathrm{MeV}\left(\frac{E_{e}}{1 \mathrm{TeV}}\right)^{2} .
$$

The charged component of these cascades is deflected by extragalactic magnetic fields (EGMF). A detailed modeling of the electromagnetic cascade process is thus not only necessary to connect the observed energy spectra of $\mathrm{TeV}$ sources with their intrinsic spectra, but provides also information about extragalactic magnetic fields (EGMF) $[2,3,4,6,5,7]$.

Another important application of electromagnetic cascades is the extragalactic diffuse gammaray background (EGRB). Since the Universe acts as a calorimeter for electromagnetic radiation, accumulating it in the $\mathrm{MeV}-\mathrm{TeV}$ range, the measured EGRB limits all processes during the history of the Universe that inject electromagnetic energy above the pair creation threshold. Examples for such processes are photo-pion and pair-production of UHECR protons with the cosmic microwave background (CMB), the decay or annihilation of (superheavy) dark matter or of topological defects [8].

I review briefly in Sec. 2 the constraints on UHECR models and the flux of cosmogenic neutrinos obtained in Ref. [9], while I discuss in Sec. 3 lower limits on the filling factor and the strength of the EGMF derived in Ref. [10].

\section{The cascade bound on UHE neutrinos}

The recently reported measurement [11] of the EGRB by Fermi-LAT, $J(E) \propto E^{-2.41}$, indicates a steeper decline with energy of the EGRB than the earlier EGRET measurement and the expectation $J(E) \propto E^{-2}$ for a cascade spectrum. We calculated in Ref. [9] the maximal energy density $\omega_{\text {cas }}$ allowed by this measurement from electromagnetic cascades both analytically and with a Monte Carlo simulation. In the former case, $\omega_{\text {cas }}$ can be calculated via

$$
\omega_{\mathrm{cas}}=\int \frac{d t d E}{1+z} E \beta_{0, \mathrm{em}}[(1+z) E] n_{p}(E, z)
$$

where $n_{p}$ is the (physical) density of protons at redshift $z, \beta_{0}(E)=(1 / E)(d E / d t)$ is the relative rate of energy loss of a proton with energy $E$ at $z=0$, and $\beta_{0, \mathrm{em}}$ denotes the relative rate of energy injected by protons into electromagnetic cascades due to pair production and pion production $\left(p \gamma \rightarrow \pi^{ \pm} \rightarrow e^{ \pm}\right.$and $\left.p \gamma \rightarrow \pi^{0} \rightarrow \gamma\right)$ at $z=0$.

The maximally allowed photon flux was determined requiring that the diffuse photon flux just touches the lower end of the error bars of the Fermi-LAT data. The corresponding bound on the 

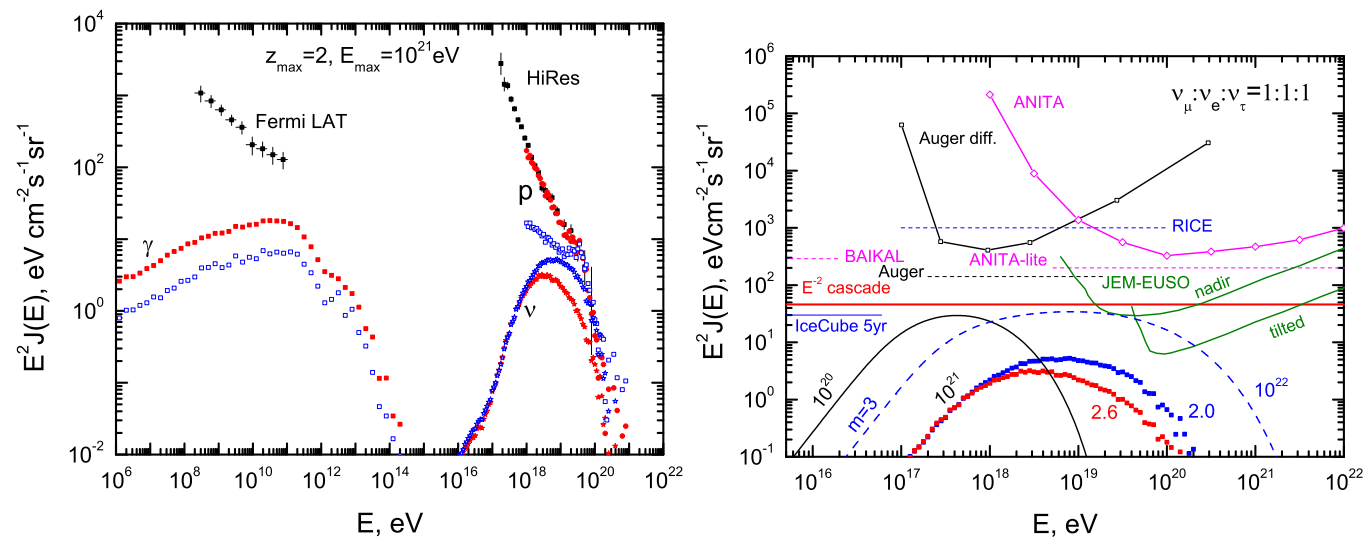

Figure 1: Left: Fermi-LAT data (black circles) for the EGRB and UHECR data from HiRes (dots) together with UHE neutrino (stars) and photon (boxes) fluxes for $E_{\max }=10^{21} \mathrm{eV}, z_{\max }=2, m=0$ and $\alpha_{g}=2.0$ (blue, open) and $\alpha_{g}=2.6$ (red, filled symbols). Right: Upper limits on the all-flavor UHE neutrino flux and expected sensitivities together with the cascade limit (" $E{ }^{-2}$ cascade"). Also shown are realistic fluxes of cosmogenic neutrinos marked by their spectral index $\alpha_{g}=2.6$ (dip model) and $\alpha_{g}=2.0$ (ankle model) together with neutrino fluxes optimized for detection by IceCube and JEM-EUSO.

cascade energy density is $\omega_{\text {cas }}^{\max }=5.8 \times 10^{-7} \mathrm{eV} / \mathrm{cm}^{3}$. This value is a factor seven smaller than the one derived earlier in Ref. [12] using the EGRET data.

In addition to the analytical treatment, we obtained the EGRB spectrum based on a Monte Carlo (MC) simulation of the cascade development. We generated CR sources from a homogeneous source distribution up to a maximal redshift $z_{\max }$. Assuming the proton injection spectrum in the form $d N / d E \propto E^{-\alpha_{g}} \vartheta\left(E-E_{\max }\right)$, we propagated the UHE protons using the Monte Carlo code described in [13], until their energy was below the threshold for $e^{+} e^{-}$pair production, $E_{\min } \approx$ $10^{18} \mathrm{eV}$, or until they reached the Earth.

We followed the evolution of electromagnetic cascades using the MC code introduced in Ref. [14] and the best-fit model of [15] for the EBL energy density. The MC procedure provides an one-dimensional description of the cascade development, taking into account the pair production and IC processes as well as adiabatic energy losses. Even EGMFs with average strengths close to the upper limit $B \sim 1 \mathrm{nG}$ have only a small influence of order $20 \%$ on the resulting EGRB.

In the left panel of Fig. 1, we show the measurement of the EGRB by Fermi-LAT [11] (black circles with error bars) together with results from our Monte Carlo simulation: The curve marked as $\alpha_{g}=2.6$ (red boxes) gives the cascade flux for the non-evolutionary ( $\left.m=0\right)$ dip model [16] with $E_{\max }=1 \times 10^{21} \mathrm{eV}$ and $z_{\max }=2$ normalized to HiRes data. The other curve marked as $\alpha_{g}=2.0$ is shown for the ankle model with a transition from galactic to extragalactic cosmic rays at $5 \times 10^{18} \mathrm{eV}$ for the same values of $E_{\max }$ and $z_{\max }$. Clearly, both models are allowed by the cascade limit.

The cascade bound is the most general bound on the UHE neutrino flux, based only on the production of electromagnetic cascades, which inevitably accompany the production of pions responsible for the neutrino flux [1]. The upper limit on the integral flux $J_{v}(>E)$ of neutrinos of all flavors is given by the following chain of inequalities,

$$
\omega_{\mathrm{cas}}^{\max }>\omega_{\mathrm{cas}}^{\pi}>\frac{4 \pi}{c} \int_{E}^{\infty} E^{\prime} J_{v}\left(E^{\prime}\right) d E^{\prime}>\frac{4 \pi}{c} E J_{v}(>E),
$$


where $\omega_{\text {cas }}^{\max }$ and $\omega_{\text {cas }}^{\pi}$ are the energy density of the cascade radiation allowed by the Fermi data and that produced only by pions, respectively. For the sake of comparison with experimental upper bounds, where a $E^{-2}$ neutrino spectrum is usually assumed, we give the upper limit for the differential cosmogenic neutrino flux of three neutrino flavors with a $E^{-2}$ spectrum and as function of the ratio of energy densities of pair- and pion-produced cascades $\omega_{\mathrm{cas}}^{e^{+} e^{-}} / \omega_{\mathrm{cas}}^{\pi}$,

$$
E^{2} J_{v}(E) \leq \frac{c}{4 \pi} \frac{\omega_{\mathrm{cas}}^{\max }}{\ln \left(E_{\mathrm{max}} / E_{\min }\right)} \frac{1}{1+\omega_{\mathrm{cas}}^{e^{+} e^{-}} / \omega_{\mathrm{cas}}^{\pi}} .
$$

This limit is plotted in the right panel of Fig. 2 as a red line labeled ' $E^{-2}$ cascade' together with existing upper limits and the expected sensitivity of IceCube and JEM-EUSO. Relaxing the $E^{-2}$ assumption, each model for cosmogenic neutrinos can be checked for consistency with the Fermi bound straightforwardly, calculating $\omega_{\text {cas }}$ via Eq. (2.1).

Choosing the parameters for the model in the lower-right corner we try to reach the sensitivity of JEM-EUSO. Since a soft spectrum increases $\omega_{\text {cas }}$, we choose the hard spectrum with $\alpha_{g}=2.0$, while $E_{\max }$ should be as large as possible. By other words we search for the extension of the ankle reference model with allowed evolution and large $E_{\max }$. We choose $E_{\max }=1 \times 10^{22} \mathrm{eV}$, with $z_{\max }=2$ and evolution parameter $m=3$. Normalized to the HiRes data, this model has $\omega_{\text {cas }}=3.3 \times 10^{-7} \mathrm{eV} / \mathrm{cm}^{3}$, i.e. is somewhat below the cascade limit. For such values, the neutrino flux is marginally detectable by JEM-EUSO.

In the lower-left corner we aim to find a model allowing IceCube the detection of cosmogenic neutrinos. Here we should increase the low-energy tail of the neutrino flux and suppress the pairproduced cascade radiation. To that end, we use $\alpha_{g}=2.0$ with strong evolution to enhance the flux of low-energy neutrinos. The maximum acceleration energy can be low, e.g. $E_{\max }=1 \times$ $10^{20} \mathrm{eV}$. Moreover, we choose evolution with $m=3.0$ and $z_{\max }=6.0$, which results in $\omega_{\text {cas }}=$ $5.5 \times 10^{-7} \mathrm{eV} / \mathrm{cm}^{3} \approx \omega_{\text {cas }}^{\max }$. As our calculations show, the flux is only marginally detectable by IceCube even for these extreme parameters.

The two models above demonstrate that even for extreme assumptions cosmogenic neutrinos remain undetectable by existing detectors such as Auger, and could be only marginally observed by IceCube and by future detectors as JEM-EUSO,

\section{Lower limits on the EGMF}

The detection of EGMFs outside clusters is crucial in discriminating different models for the origin of their seed fields, but extremely challenging using traditional methods as e.g. Faraday rotation. An alternative approach to obtain information about the EGMFs is to use its effect on the radiation from $\mathrm{TeV}$ gamma-ray sources. Potentially observable effects of such electromagnetic cascades in the EGMF include the delayed "echoes" of multi-TeV $\gamma$-ray flares $[3,6]$ and the appearance of extended emission around initially point-like $\gamma$-ray sources [2, 4, 5, 7].

An additional way to derive lower limits on the EGMF has been pointed out recently $[17,18]$ : Since the deflection of the cascade flux into an extended halo weakens the point-like image, the non-observation of $\mathrm{TeV}$ blazars in the $\mathrm{GeV}$ range by Fermi-LAT can been used to derive a lower limit on the EGMF. Particular suitable candidates are blazars with a very hard TeV spectrum like 

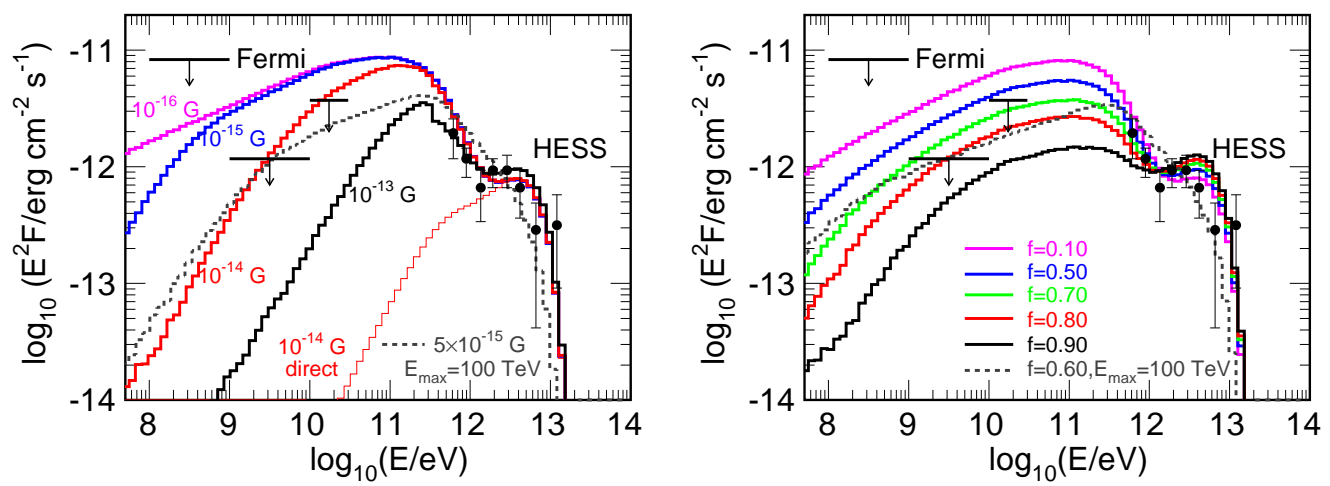

Figure 2: Left: Fluence contained inside the 95\% confidence contour of the PSF of Fermi-LAT as function of energy together with Fermi-LAT upper limits and HESS observations for a uniform EGMF with strengths varying from $B=10^{-16} \mathrm{G}$ to $B=10^{-13} \mathrm{G}$ with $E_{\max }=20 \mathrm{TeV}$ (solid) and $100 \mathrm{TeV}$ (dashed). The direct component for $B=10^{-14} \mathrm{G}$ is also shown. Right: Fluence contained inside the $95 \%$ confidence contour of the PSF of Fermi-LAT as function of energy for a EGMF with top-hat profile and filling factor $f$ varying from $f=0.1$ to $f=0.9$ with $E_{\max }=20 \mathrm{TeV}$ (solid) and $100 \mathrm{TeV}$ (dashed).

1ES $0229+200$ that show a low intrinsic GeV emission. In this way, [17] and [18] derived the lower bound $B \gtrsim 5 \times 10^{-15} \mathrm{G}$ on the EGMF.

We improved in Ref. [10] on these previous analyses in two respects: First, we used a Monte Carlo simulation for the development of electromagnetic cascades in the EBL that includes the effects of magnetic fields like synchrotron radiation and deflections of electrons. Second, we examined the influence of a more realistic, structured magnetic field on the EGMF limit.

In the two panels of Fig. 2 we show our results for the fluence contained inside the 95\% confidence contour of the PSF of Fermi-LAT. Additionally, these figures contain the HESS observations as black dots with error bars and the Fermi-LAT upper limits derived by [18]. The fluences have been normalized fitting them to the HESS data.

In the left panel, we used a uniform magnetic field so that our results can be directly compared with the analytical estimates of [18]. Note that a turbulent field with correlation length $L_{\mathrm{cr}}$ much larger than the mean free path $l_{\mathrm{IC}}$ of electrons in the Thomson regime, $L_{\mathrm{cr}} \gg l_{\mathrm{IC}} \sim 5 \mathrm{kpc}$, is well approximated by a uniform field. For smaller correlation lengths, $L_{\mathrm{cr}} \ll l_{\mathrm{IC}}$, the electron diffuses in the small-angle deflection regime, requiring larger magnetic fields for the same deflection angle. Demanding that the cascade flux is below the upper limits of Fermi-LAT leads to a lower limit on the magnetic field strength of $\sim 10^{-14} \mathrm{G}$. For this case the direct component, i.e. photons arriving at the detector without cascading, is also shown. Note that for small $E_{\max }$ the transition from the direct to the cascade contribution leads to a break at $\sim \mathrm{TeV}$ in the spectrum, as suggested by the HESS data.

While our limit agrees reasonably well with the analytical estimate of [18], the shape of the cascade flux obtained differs. There are several reasons responsible for these differences: First, [18] assume that the spectral shape of the cascade flux below the threshold energy $\sim 10^{11} \mathrm{eV}$ is given by $\mathscr{F} \propto E^{0.5}$ for negligible magnetic fields. Such a slope typical for the regime of Thomson cooling is however restricted to energies $E \lesssim 10^{8} \mathrm{eV}$, while at higher energies a plateau $\mathscr{F} \propto E^{\alpha}$ with $\alpha \sim 0.9$ is expected [9]. Second, deflections in the EGMF lead even for isotropic emission 
to extended images of point-like sources. This effect has been neglected in [18]. Third, using full probability distributions for the interactions there is a non-negligible probability for photons not to interact, especially towards the low-energy end of the injected energy range. Finally, the energy dependent PSF of Fermi introduces an artificial energy dependence of the point-like flux $\mathscr{F} \propto \vartheta_{95}^{2}$. Note that an increase in $E_{\max }$ from 20 to $100 \mathrm{TeV}$ reduces the limit on the magnetic field strength to $\sim 5 \times 10^{-15} \mathrm{G}$, see Fig. 1, while a further increase of $E_{\max }$ strengthens the limit again. The counter-intuitive behavior between 20 to $100 \mathrm{TeV}$ is caused by the dominance of direct photons at the high-energy part of the spectrum for small $E_{\max }$.

Since the EGMF is strongly structured, one may wonder how a non-uniform field modifies this limit. In particular, we want to address the question whether the presence of relatively strong fields concentrated inside cosmic structures like filaments could mimic the effect of an EGMF present also in voids. As simplest possible test, we use first a top-hat profile for the structure of the EGMF: We set the field strength to zero in a fraction $1-f$ of space and use a value which in general is assumed to be representative for filaments, $B=10^{-10} \mathrm{G}$, in the remaining part. For the separation of the peaks we use $D=10 \mathrm{Mpc}$ motivated by the typical distances between cosmological structures, although the exact value of $D$ plays no role as long as $(1-f) D \ll l_{\gamma}$, where $l_{\gamma}$ denotes the mean free path of photons. The dependence of the fluence contained inside the PSF of Fermi-LAT on the filling factor $f$ is shown in the right panel. To be consistent with the Fermi upper limits, sufficiently strong magnetic fields should fill $\gtrsim 80 \%$ of space. The derived limit on the filling factor is practically independent of $B$, as long as the field is stronger than $\gtrsim 5 \times 10^{-15} \mathrm{G}$. As in the previous case, by assuming a higher injected $E_{\max }$ the required filling factor is slightly reduced to $60 \%$.

The failure of strong fields filling only a small fraction of the universe to suppress sufficiently the point-like cascade flux can be understood as follows: The HESS observations of 1ES 0229+200 cover the energy range $0.5-11 \mathrm{TeV}$. In the same energy range, the mean free path $l_{\gamma}$ of VHE $\gamma$-rays through the EBL varies between 1000 and $50 \mathrm{Mpc}$ and is thus always much larger than the typical extension of regions with large fields, $(1-f) D$. For the energies considered, the cascade consists typically of only three steps, $\gamma \rightarrow e^{ \pm} \rightarrow \gamma$. Since the mean free path $l_{\mathrm{IC}}$ of electrons in the Thomson regime is very small, $l_{\mathrm{IC}} \sim 1 \mathrm{kpc}$, all cascades with electrons created outside the strong-field regions are undeflected. Thus it is not possible to trade smaller values of $f$ against larger values of $B$ : Increasing the field strength beyond $\sim 10^{-13} \mathrm{G}$ leads only to an increase of the deflection, while the fraction of cascades deflected outside the Fermi PSF remains constant.

\section{Summary}

I have reported on a calculation of the fluence of 1ES 0229+200 as seen by Fermi-LAT using a Monte Carlo simulation for the cascade development. Since the electron cooling length is much smaller than the mean free path of the $\mathrm{TeV}$ photons, a sufficient suppression of the point-like flux requires that the EGMF fills a large fraction along the line-of-sight towards 1ES 0229+200, $f \gtrsim 0.6$. The lower limit on the magnetic field strength in this volume is $\sim 5 \times 10^{-15} \mathrm{G}$ for a stationary and or $\sim 10^{-17} \mathrm{G}$ for flaring source, respectively. This limit puts very stringent constraints on the origin of EGMFs: Either the seeds for EGMFs have to be produced by a volume filling process (e.g. primordial) or very efficient transport processes have to be present which redistribute mag- 
netic fields that were generated locally (e.g. in galaxies) into filaments and voids with a significant volume filling factor.

I have also reported on recently derived constraints on models for UHECR and cosmogenic UHE neutrinos which demonstrate that the latter are not detectable with the present experimental sensitivity. Both the dip and ankle model without or with weak evolution are consistent with the Fermi-LAT measurement of the EGRB. The cosmogenic neutrino flux is strongly limited by the new upper cascade bound and undetectable for a conservative choice of parameters by Auger-North and JEM-EUSO. Only for an extreme set of parameters, $E_{\max } \gtrsim 1 \times 10^{22} \mathrm{eV}$ and $\omega_{\text {cas }} \sim \omega_{\text {cas }}^{\max }$, the cosmogenic flux is marginally detectable by JEM-EUSO. To achieve the observation of cosmogenic neutrinos for less extreme parameters, the detection threshold of JEM-EUSO (in the tilted mode) must be lowered down to $1 \times 10^{19} \mathrm{eV}$ and the sensitivity of Auger-North should be increased by factor $\sim 20$ in comparison with Auger-South. The results of our paper emphasize the necessity to develop more sensitive methods as e.g. radio-detection for the detection of cosmogenic neutrinos.

\section{Acknowledgments}

I am grateful to V. Berezinsky, K. Dolag, A. Gazizov, S. Ostapchenko and R. Tomàs for fruitful collaborations on which this presentation is based on and to Norsk Forsknigsradet for support.

\section{References}

[1] V. S. Berezinsky and A. Yu. Smirnov, Astrophys. Sp. Sci. 32461 (1975);

[2] F. A. Aharonian, P. S. Coppi, and H. J. Völk, Astrophys. J. 423, L5 (1994).

[3] R. Plaga, Nature, 374, 430 (1995).

[4] A. Neronov and D. V. Semikoz, JETP Lett. 85, 473 (2007).

[5] K. Dolag, M. Kachelrieß, S. Ostapchenko and R. Tomàs, Astrophys. J. 703, 1078 (2009).

[6] K. Murase et al., Astrophys. J. 686, L67 (2008).

[7] A. Neronov et al., Astrophys. J. 719, L130 (2010).

[8] M. Kachelrieß, Comptes Rendus Physique 5, 441 (2004).

[9] V. Berezinsky, A. Gazizov, M. Kachelrieß and S. Ostapchenko, Phys. Lett. B 695, 13 (2011).

[10] K. Dolag, M. Kachelrieß, S. Ostapchenko and R. Tomàs, Astrophys. J. 727, L4 (2011).

[11] A. A. Abdo et al. [Fermi-LAT collaboration], Phys. Rev. Lett. 104, 101101 (2010).

[12] R. Aloisio, V. Berezinsky and M. Kachelrieß, Phys. Rev. D 69, 094023 (2004).

[13] M. Kachelrieß and D. Semikoz, Astropart. Phys. 23, 486 (2005).

[14] M. Kachelrieß, S. Ostapchenko and R. Tomàs, New J. Phys. 11, 065017 (2009).

[15] T. M. Kneiske and H. Dole, Astron. Astrophys. 515, id.A19 (2010).

[16] V. Berezinsky, A. Z. Gazizov and S. I. Grigorieva, Phys. Rev. D 74, 043005 (2006); astro-ph/0210095.

[17] A. Neronov and I. Vovk, Science 328, 73 (2010).

[18] F. Tavecchio et al., Mon. Not. Roy. Astron. Soc. 406, L70 (2010). 